\title{
Cancer Growth Inhibition Using Predictive Mathematical Models of Signaling Pathways
}

\author{
Aadil Rashid Sheergojri, Pervaiz lqbal and Ashiq Mohd llyas
}

\begin{abstract}
Department of Mathematics and Actuarial Science, B. S. Abdur Rahman Crescent Institute of Science and Technology Chennai, India
\end{abstract}

\begin{abstract}
Cancer cells develop several hallmark changes over the progress of the tumor process. Cell assistance in multicellular organisms is regulated by the division of cell coordination by aggressive growth modulation. In this perspective, the use of molecular indicators triggering cell division is a mystery, because a cancer cell can manipulate any molecule that induces and helps growth, disturbing cellular assistance. An effective alteration proceeding to tumors must develop to be competitive, allowing a cancer cell to pass a signal resulting in better selection chances. The subjective simulation of physiological systems has become increasingly valuable in recent years, and there is now a wide range of mathematical models of signalling pathways that have contributed to some groundbreaking discoveries and hypotheses as to how this system works. Here we discuss various modeling methods and their application to the physiology of medical systems, focusing on the identification of parameters in ordinary differential equation models and their significance for forecasting cellular decisions in network modeling. In situations of global and local cell-to-cell rivalry, we quantify how this mechanism impacts a mutated cell's fixing chance of producing such a signal, and consider that this process will play a vital role in reducing cancer.
\end{abstract}

Keywords: Cancer Growth, Modeling, Signaling Pathways, Predictive Mathematical Models.

\section{INTRODUCTION}

The cells of the human body are the fundamental and operational components of life. Healthy cells may expand, differentiate or probably die to create new cells. This mechanism will be the natural process for cells, and cells develop faster enabling the individual to expand, particularly in the early stages of our lives. Owing to the behavior of the substances involved in various biological processes, cells experience changes [1]. The significant function of every biological system is its capacity to interact with that of the natural world. Such knowledge sharing is called cell signaling and is dependent on the cell's ability to provide appropriate responses to external stimuli. A dynamic network of connections conducts cellular signals. In this system, various pathways of communication can be identified consisting of several concurrent activities, involving association between protein and protein, receptor modification, Post-translational alteration and partitioning $[2,3]$.

Signaling pathways help cells to identify patterns in their surroundings, absorb explicit or implicit signals, and react upon them by shifts in cytokine behavior, morphology, and/or regulatory changes [4]. Knowing the structure and geometry of protein and lipid kinase-driven signaling networks is essential in rationalizing the structural foundation of signal modulation and how this mechanism is manipulated to disorder. An identified solution to the geometry and connectivity of kinase networks includes the need for rigorous history-mining techniques to compile lists on the interactions among proteins in signaling flows [5-7].

*Address correspondence to this author at the Department of Mathematics and Actuarial Science, B. S. Abdur Rahman Crescent Institute of Science and Technology Chennai, India; E-mail: pervaizmaths@gmail.com
Microbiologists and cellular biologists sometimes wonder why we need computational models to recognize biochemical procedures. Furthermore, related to the nature of biochemical processes, it is obvious that even a higher degree of interpretation is needed to decipher the 'terminology' of cells and obtain perspectives of how environmental signals are incorporated and how reactions are controlled for differentiation or division, life, and mortality [8]. Signaling network computational models explain the spatial and temporal development of some symbolic elements of the signaling network, known as "nodes," such that their interactions or "networking" are known to one another. Those nodes may reflect subspecies of proteins and varying biomolecular modifications in their relations. The routing algorithms are comprised of the networks and the links that bind them together [2].

From its inception cancer control has been a big threat to multicellularity. It is important especially in mammals, whereby cells are versatile and can disperse across the multicellular organism. The several pathways emerging to combat this multiplication demonstrate that cancer imposes tremendous developmental stress on the organisms. Those pathways will consist of the immune system and related tumor antigens like tumor protein P53, also known as p53, cellular tumor antigen p53, cell growth-limiting factors like telomere narrowing, and tumor suppressor genes like Adenomatous polyposis coli (APC) $[9,10]$. It has also been argued that cellular development, and in particular tissues under-regulated cell production, is being developed to fight cancer. Ultimately, the multi-stage cancer hypothesis notes that a tumor takes many alterations in becoming cancerous, indicating that species are screened against cancer where only a few alterations will contribute. 
Signaling substances that cause cell growth is an illustration of the growth factors. They are released between the cell spaces and can then spread both to the cell that created it, and also to other cells in the surrounding. When the signal is unfavorable to the sender, another cell may be out-competing the signaller allele. Here, we concentrate on signals which may support cancer and analyze the value and distribution of these signals. The pathways to avoid cancer development are to control the production of some genes by an effective diffusive signal, for instance, a growth factor. A different collection of genes may display a similar pattern of transcription to achieve an impact comparable to the behavior of the diffusive signal. The Multistage Hypothesis indicates that this requires a mysterious approach that is hard to develop, in which multiple alterations are needed to create a common trend of gene regulation requiring a series of phases, namely interphase (G1), prophase (G2), metaphase (G3), anaphase (G4), telophase (G5) and cytokinesis (G6) to change the transcription within each gene, activating unregulated cell growth [11-14].

Here, we will explain the modeling of signaling pathways along with some of the difficulties we find available in modeling those pathways and to illustrate the importance of modeling methods in solving urgent biological problems, we offer an outline of modeling principles explaining biological processes, in specific cancer signaling pathways. We further stress that by using predictive models to forecast cellular activity, some modeling technique criteria have to be fulfilled to promote the prediction of cellular activity and a better knowledge of the living cell. We believe that signaling within cells of multicellular species shows mechanisms that may contribute to a decrease in the risk of cancer growing. We demonstrate in general that these cancer protections would be present if the signals that could support cancer cells are effective to have a broad area of impact. By defining our model, we will begin through the different iterations evaluated-cumulative gain of obtaining the signal from several cells or un-cumulative, one- and two-dimensional chambers, and various propagation and comparison levels.

\section{METHODOLOGY}

The influence of alteration was examined, which results in a development in the distribution of an effective diffusive agent that activates cell differentiation (growth factor). These agents can influence the cell which created it explicitly or implicitly, and also the cells throughout the surrounding. The rise throughout the frequency of dividing cells is signified as an advantage in the model.
Two forms of selections have been regarded: Some cells throughout the system will be selected for mortality in the overall selection after a cell is selected for development. This example reflects the situation when compartmental spatial and asset activity in a system will not allow separation into consideration. A cell inside a specific range of the replicating cells is selected for mortality in regional selection.

\section{Randomness and the Fixation Probability}

The amount of signaling cells, $T_{S}=j$, may either be raised by one $(j+1)$, remain at the same $(j)$ or reduced by one $(j-1)$ at every time stage.The probability for transformation from $\mathrm{j}$ to $(j+1)$ and $(\mathrm{j}-1)$ will be given by:

$$
\begin{aligned}
& P_{j, j+1}=R_{R} Q_{N} \\
& P_{j, j-1}=R_{N} Q_{R} \\
& P_{j, j}=1-P_{j, j+1}-P_{j, j-1}
\end{aligned}
$$

Here $R_{R}$ and $R_{N}$ are possibilities of being selected for reproduction by the signaling and non-signaling cells and $Q_{N}$ and $Q_{R}$ indicate the probability of the signaling and non-signaling cells being selected for mortality, respectively.

For the two absorbent states $\mathrm{j}=0$ or $\mathrm{j}=\mathrm{N}$, remember $\mathrm{N}$ need not be the size of the organism for our function. It seems that all the cells in the organism are healthy, so we're open to the idea of evolving cancer exceeding any crucial size $\mathrm{N}$ of the carrying capacity of the tumor. We're concerned about the probability of hitting $\mathrm{T}_{\mathrm{S}}=\mathrm{N}$ when starting from condition $\mathrm{j}$. To determine the likelihood of $\mathrm{j}$ defective cells eventually taking over the species, we find that this likelihood of fixation $b_{j}$ fits the equation:

$b_{j}=P_{j, j+1} b_{j+1}+P_{j, j} b_{j}-P_{j, j-1} b_{j-1}$

For the limiting conditions $\mathrm{b}_{0}=0$ and $\mathrm{b}_{\mathrm{N}}=1$.

Iteration of this equation results in a solution:

$\mathrm{b}_{\mathrm{j}}=\frac{1+\sum_{\mathrm{i}=1}^{\mathrm{j}-1} \prod_{\mathrm{k}=1}^{\mathrm{i}} \mathrm{P}_{k, \mathrm{k}-1} / \mathrm{P}_{\mathrm{k}, \mathrm{k}+1}}{1+\sum_{\mathrm{i}=1}^{\mathrm{N}-1} \prod_{\mathrm{k}=1}^{\mathrm{i}} \mathrm{P}_{\mathrm{k}, \mathrm{k}-1} / \mathrm{P}_{\mathrm{k}, \mathrm{k}+1}}$

See e.g., [15] for details of the derivation and here $\mathrm{P}_{\mathrm{k}, \mathrm{k}+1}$ and $\mathrm{P}_{\mathrm{k}, \mathrm{k}-1}$ are specified in equations (1) and (2) for randomness respectivelyand $k=0,1,2, \ldots n$.

For a single signaling cell, the fixation probability $\varphi_{\mathrm{S}}\left(=\mathrm{b}_{1}\right)$ is therefore

$\varphi_{\mathrm{S}}=\mathrm{b}_{1}=\frac{1}{1+\sum_{\mathrm{i}=1}^{\mathrm{N}-1} \prod_{\mathrm{k}=1}^{\mathrm{i}} \frac{\mathrm{P}_{\mathrm{k}, \mathrm{k}}-1}{\mathrm{P}_{\mathrm{k}, \mathrm{k}}+1}}$ 
We get a well-known equation, when $P_{k, k-1} / P_{k, k+1}$ is fixed, as $(1+\mathrm{S})^{-1}$

$\varphi_{\mathrm{S}}=\mathrm{b}_{1}=\frac{1}{1+\sum_{\mathrm{i}=1}^{\mathrm{N}-1}(1+\mathrm{S})^{-\mathrm{i}}}=\frac{1-(1+\mathrm{S})^{-1}}{1-(1+\mathrm{S})^{-\mathrm{N}}}$

This helps us to quantify the signaling cell probability of achieving size $\mathrm{N}$.

\section{Directional Flows in Biological Processes}

In the context of biological processes, two separate strategies have emerged to decipher dynamic signaling pathways or their fundamental governing processes. The 'top-down' method integrates huge-scale 'omics' data involving subjective and fixed cellular portion knowledge with genomics methods for evaluating routing protocols. Another method, 'bottom-up,' produces small to intermediate throughput data with econometric input material that is important for the calibration of highly predictive capacity moderately sized predictive models. The major problem in biological processes is to incorporate all methods to obtain broad computational network models that are sufficiently complex to decode governing processes and forecast metabolic activity reliably, and also the possible effect of clinical action.

\section{Mathematical Structures Tackle Uncertainty at Various Stages}

To explain and analyze biological processes at specific levels, various mathematical techniques were implemented and optimized. A cellular process described by Ordinary Differential Equations (ODEs) has been the highest accepted method for describing cellular reactions and modifications of signaling networks in the mathematical method.ODE systems typically reflect the response levels (i.e. interaction, dissociation, development, and deterioration of organisms) because of the kinetic scheme for chemical reaction networks which says that the rate of a chemical reaction is proportional to the product of the concentrations of the reacting chemical species known as mass action kinetics. For instance, the estimation of Michaelis - Menten is obtained via ODEs for mass action and implemented in enzyme kinetics, as ODE systems can combine significant and time-resolved details, such systems permit the understanding and interpretation of dynamic behaviors that might contribute to non-intuitive findings within biological processes.

\section{DISCUSSION}

In this study we examined the situations whereby a defective cell can infect a production of cells, releasing a substance that promotes cell development. We have seen that if the molecule is effective to make, and the advantage is offered not only to the signaling cell and to sufficiently adjacent cells, the mutant's attack can be avoided. In addition, we showed that this effect is intensified when selection among cells is local, meaning that even though the gain is only granted to a limited cell count across the signaler, the mutant attack is prevented.

Our theory indicates that the effectiveness and distribution of signaling in multicellular species can influence the probability of cancer arising, so an adaptive regulation of both can reduce cancer occurrence. Many processes appear to have developed to resist cancer through regulation of the immune system, mortality of cells, cell multiplication limit, to development of tissues[12, 15]. Here some signaling predictions for living organisms have been considered and we argue that signals that may help a cancer cell may inflict, continuing to disperse or affect other cells across the signaler. These processes should be more efficient for local rivalry in tissues. Thus, such processes should not be assumed to be successful throughout the body. Organs of less rivalry will continue to provide more cancer changes associated with either the signaling or growth factor and organs with greater rivalry will adapt by eliminating the signal.

Current aims in the hybrid method of results-driven mathematical modeling are the incorporation of several collaborating and alleviating signaling mechanisms into broad network systems that were extensively optimized with subjective details. Predictive mechanisms only then are developed with recognizable conditions capable of simulating empirically non-observable cell behavior, identifying regulatory pathways and predicting cellular drug reactions. Developments in mathematical techniques, computing resources, and advanced technology are needed to accomplish these objectives.

\section{ACKNOWLEDGEMENT}

The author acknowledges our esteemed institution for giving us the monthly fellowship.

\section{DECLARATIONS}

\section{Conflict of Interest}

The authors declare that they have no conflict of interest.

\section{REFERENCES}

[1] Stamouli S. Mathematical modeling of normal and cancer prostate signaling pathways [dissertation]. Uppsala, Sweden: Uppsala universitet 2015. 
[2] Ventura AC, Jackson TL, Merajver SD. On the role of cell signaling models in cancer research. Cancer Res 2009; 69(2): 400-2.

https://doi.org/10.1158/0008-5472.CAN-08-4422

[3] Kholodenko BN. Cell-signalling dynamics in time and space. Nat Rev Mol Cell Biol 2006; 7(3): 165-76. https://doi.org/10.1038/nrm1838

[4] Klipp E, Liebermeister W. Mathematical modeling of intracellular signaling pathways. BMC Neurosci 2006; 7(1): 1-6. https://doi.org/10.1186/1471-2202-7-S1-S10

[5] Hijazi M, Smith R, Rajeeve V, Bessant C, Cutillas PR. Reconstructing kinase network topologies from phosphoproteomics data reveals cancer-associated rewiring. Nat Biotechnol 2020; 38(4): 493-502.

https://doi.org/10.1038/s41587-019-0391-9

[6] Hanahan D, Weinberg RA. Hallmarks of cancer: the next generation. Cell 2011; 144(5): 646-74. https://doi.org/10.1016/j.cell.2011.02.013

[7] Kanehisa M, Furumichi M, Tanabe M, Sato Y, Morishima K. KEGG: new perspectives on genomes, pathways, diseases and drugs. Nucleic Acids Res 2017; 45(D1): D353-61. https://doi.org/10.1093/nar/gkw1092

[8] Bachmann J, Raue A, Schilling M, Becker V, Timmer J, Klingmüller $U$. Predictive mathematical models of cancer signalling pathways. J Intern Med 2012; 271(2): 155-65. https://doi.org/10.1111/j.1365-2796.2011.02492.x
[9] Oña L, Lachmann M. Signalling architectures can prevent cancer evolution. Sci Rep 2020; 10(1): 1-9. https://doi.org/10.1038/s41598-020-57494-w

[10] Roake CM, Artandi SE. Control of cellular aging, tissue function, and cancer by p53 downstream of telomeres. Cold Spring Harb Perspect Med 2017; 7(5): a026088. https://doi.org/10.1101/cshperspect.a026088

[11] Iwasa Y, Michor F, Nowak MA. Evolutionary dynamics of invasion and escape. J Theor Biol 2004; 226(2): 205-14. https://doi.org/10.1016/j.jtbi.2003.08.014

[12] Archetti M. Evolutionarily stable anti-cancer therapies by autologous cell defection. Evol Med Public Health 2013; 2013(1): 161-72. https://doi.org/10.1093/emph/eot014

[13] Caulin AF, Maley CC. Peto's Paradox: evolution's prescription for cancer prevention. Trends Ecol Evol 2011; 26(4): 175-82. https://doi.org/10.1016/j.tree.2011.01.002

[14] Antal T, Scheuring I. Fixation of strategies for an evolutionary game in finite populations. Bull Math Biol 2006; 68(8): 1923-44.

https://doi.org/10.1007/s11538-006-9061-4

[15] Archetti M, Ferraro DA, Christofori G. Heterogeneity for IGF-II production maintained by public goods dynamics in neuroendocrine pancreatic cancer. PNAS 2015; 112(6): 1833-8.

https://doi.org/10.1073/pnas.1414653112

https://doi.org/10.6000/1929-6029.2021.10.12

(C) 2021 Sheergojri et al.; Licensee Lifescience Global.

This is an open access article licensed under the terms of the Creative Commons Attribution License (http://creativecommons.org/licenses/by/4.0/) which permits unrestricted use, distribution and reproduction in any medium, provided the work is properly cited. 